\title{
Longitudinal thermalization via the chromo-Weibel instability
}

\author{
M. Attems ${ }^{*}{ }^{a b}$ A. Rebhan ${ }^{a}$, and M. Strickland ${ }^{b c}$ \\ ${ }^{a}$ Institut für Theoretische Physik, Technische Universität Wien \\ Wiedner Hauptstrasse 8-10, 1040 Vienna, Austria. \\ ${ }^{b}$ Frankfurt Institute for Advanced Studies \\ Ruth-Moufang-Str. 1, 60438 Frankfurt am Main, Germany. \\ ${ }^{c}$ Department of Physics, Kent State University \\ Kent, $\mathrm{OH} 44242$, USA.
}

\begin{abstract}
Non-Abelian plasma instabilities play an important role in the non-equilibrium dynamics of a weakly coupled quark-gluon plasma. Using the discretized hard loop framework we calculate the time evolution of soft gluonic fields in a longitudinally free streaming background. Extrapolating our results to energies probed in relativistic heavy-ion collisions we find a pressure anisotropy that persists for a few fm/c. However, the chromofields quickly develop a Boltzmann longitudinal energy spectrum, suggesting fast longitudinal thermalization of the quark gluon plasma even though it remains momentum-space anisotropic. In this proceedings contribution we review our recent numerical results, present new results for the scaling of the isotropization time with the initial current fluctuation amplitude, and present tests of the gauge invariance of the extracted longitudinal spectra.
\end{abstract}

PACS: 11.15Bt, $04.25 . \mathrm{Nx}, 11.10 \mathrm{Wx}, 12.38 \mathrm{Mh}$

Xth Quark Confinement and the Hadron Spectrum,

October 8-12, 2012

Technische Universität München, Campus Garching, Munich, Germany

\footnotetext{
* Speaker.
} 


\section{Introduction}

The determination of the thermalization and isotropization time a quark gluon plasma (QGP) produced in relativistic heavy ion collisions is an important open question. In this proceedings contribution we review recent numerical simulations which use the hard-expanding-loop framework for describing the evolution of non-Abelian plasma instabilities in a free-streaming background [1] where we observed the appearance of a Boltzmann distribution of the energy in longitudinal wave number. In addition, we present two extensions of previous results: (a) results on the scaling of the plasma isotropization time with the initial current fluctuation amplitude and (b) test the gauge invariance of the extracted longitudinal spectra subject to random local gauge transformations. We find that the isotropization time scales with $\left(\log \Delta^{-1}\right)^{2}$ with $\Delta$ being the initial current fluctuation amplitude. The quasi-thermal longitudinal energy spectra, while being inherently gauge dependent, turn out to be reasonably robust against random gauge transformations as long as the latter do not introduce uncomfortably large numerical errors in the gauge invariant sum over the energy modes.

\section{The Hard Expanding Loop Framework}

The investigation of the evolution of soft (gauge) fields subject to dynamical instabilities such as the chromo-Weibel instability [2-14] is an active area of research. Field dynamics in an expanding background have been recently investigated using classical Yang-Mills simulations [15 - 18], analytically in the high energy limit $[19,20]$, within scalar $\phi^{4}$ theory subject to parametric resonance instabilities [21], and SU(2) Vlasov-Yang-Mills [22, 23, 1] including longitudinal expansion. In this contribution we will report on the last category of investigations, namely those performed using SU(2) Vlasov-Yang-Mills simulations in a longitudinally free-streaming background. The simulations using this framework rely on the hard-loop approximation which is valid at asymptotically weak coupling and provide information on the evolution of the quark gluon plasma in the high energy limit. The resulting analytical and numerical framework has been dubbed the hard expanding loop (HEL) framework. Below we briefly summarize the key points relevant for such studies and refer the reader to Ref. [1] for more details.

In this framework we assume that the background particles are longitudinally free streaming and as a result the background (hard) particles possess a local rest frame momentum-space anisotropy which increases monotonically in proper-time as $\xi(\tau)=\left(\tau / \tau_{\text {iso }}\right)^{2}-1$, where $\tau_{\text {iso }}$ corresponds to the proper time at which the distribution is originally isotropic (when $\tau_{\text {iso }}<\tau_{0}$ with $\tau_{0} \sim Q_{s}^{-1}$, the hard particle distribution starts already anisotropic). With any isotropic distribution $f_{\text {iso }}$, the free streaming background distribution, $f_{0}$, is constructed as

$$
f_{0}(\mathbf{p}, x)=f_{\text {iso }}\left(\sqrt{p_{\perp}^{2}+\left(\frac{p^{\prime z} \tau}{\tau_{\text {iso }}}\right)^{2}}\right)=f_{\text {iso }}\left(\sqrt{p_{\perp}^{2}+p_{\eta}^{2} / \tau_{\text {iso }}^{2}}\right) .
$$

Following [1] we begin with the Vlasov-type dynamical equation which is obeyed by color perturbations $\delta f^{a}$ of a color-neutral longitudinally free-streaming expanding momenta distribution $f_{0}$

$$
\left.V \cdot D \delta f^{a}\right|_{p^{\mu}}=g V^{\mu} F_{\mu \nu}^{a} \partial_{(p)}^{v} f_{0}\left(\mathbf{p}_{\perp}, p_{\eta}\right) .
$$


Eq. (2.2) has to be solved simultaneously with the non-Abelian Yang-Mills equations which couple the color-charge fluctuations back to the gauge fields via the induced color-currents $j_{a}^{v}$

$$
D_{\mu} F_{a}^{\mu v}=j_{a}^{v}=g t_{R} \int \frac{d^{3} p}{(2 \pi)^{3}} \frac{p^{\mu}}{2 p^{0}} \delta f_{a}(\mathbf{p}, \mathbf{x}, t) .
$$

The above equations are then transformed to comoving coordinates with the metric $d s^{2}=d \tau^{2}-$ $d \mathbf{x}_{\perp}^{2}-\tau^{2} d \eta^{2}=g_{\alpha \beta} d x^{\alpha} d x^{\beta}$. In these coordinates the four-velocity $V^{\alpha}$ of hard particles has the form

$$
V^{\alpha}=\frac{p^{\alpha}}{p_{\perp}}=\left(\cosh (y-\eta), \cos \phi, \sin \phi, \frac{1}{\tau} \sinh (y-\eta)\right)
$$

and, as usual, $D_{\alpha}=\partial_{\alpha}-i g\left[A_{\alpha}, \cdot\right]$ is the gauge covariant derivative and $F_{\alpha \beta}=\partial_{\alpha} A_{\beta}-\partial_{\beta} A_{\alpha}-$ $i g\left[A_{\alpha}, A_{\beta}\right]$ is the field strength tensor with strong coupling $g$. To numerically solve the resulting set of coupled non-linear partial differential equations, which are expressed compactly via Eqs. (2.2) and (2.3), we use temporal gauge $A^{\tau}=0$ and introduce gauge-field canonical momenta

$$
\Pi^{i}=\tau \partial_{\tau} A_{i} \quad \Pi^{\eta}=\frac{1}{\tau} \partial_{\tau} A_{\eta} .
$$

The canonical momenta are related to the transverse and longitudinal chromoelectric field components via $E_{i}=\Pi_{i} / \tau$ and $E_{L}=\Pi^{\eta}$, respectively. One can express the equations of motion in terms of canonical momenta $\Pi$ and gauge links $U$ with $U_{\mu}$ being the parallel transporter in $\mu$ direction:

$$
U_{i}=\exp \left(-i A^{i}\right) \quad U_{\eta}=\exp \left(i a_{\eta} A_{\eta}\right) .
$$

In addition, one introduces auxiliary fields $\mathscr{W}(\tau, x ; \phi, y)$ which describe the induced current fluctuations at each point $x$ of the three-dimensional space at given proper time $\tau$. At each space-time point these auxiliary fields also depend on the light-like velocity of hard particles specified by the azimuthal angle $\phi$ and momentum-space rapidity $y$. For details we refer the reader to Ref. [1].

\section{Initial Current Fluctuations}

One of the key questions which we wish to address with HEL simulations is the question of how long does it take for the system to become isotropic in momentum space when particle and field contributions are combined. Due to the fact that the hard particles are assumed to be undergoing longitudinal free-streaming, they will have a longitudinal pressure which decreases asymptotically as $\tau^{-3}$ and a transverse pressure which decreases asymptotically as $\tau^{-1}$. In response to this growing momentum space anisotropy unstable chromofields grow rapidly after some initial transient period which depends, in part, on details of the initial spectrum. At late times, one finds that the field pressures become large and are able to completely compensate for the anisotropy present in the particle sector. As a result, at some point in time, the total (particle + field) pressure will be become isotropic with $\mathscr{P}_{T}=\mathscr{P}_{L}$. One can extract this time scale directly from the simulations and, as shown in Ref. [1], one finds that the resulting isotropization time-scale $\tau_{\text {ISO }}$ (not to be confused with the initial $\tau_{\text {iso }}$ ) primarily depends on the initial current fluctuation amplitude $\Delta$ used as a seed as specified in Ref. [24]. 
(a)

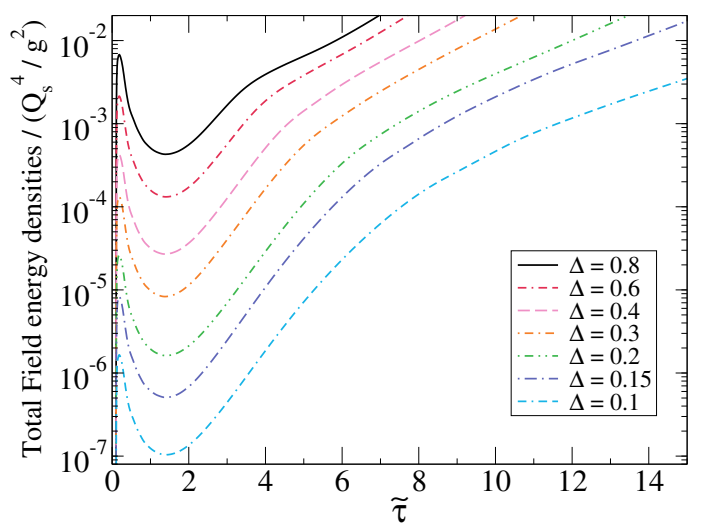

(b)

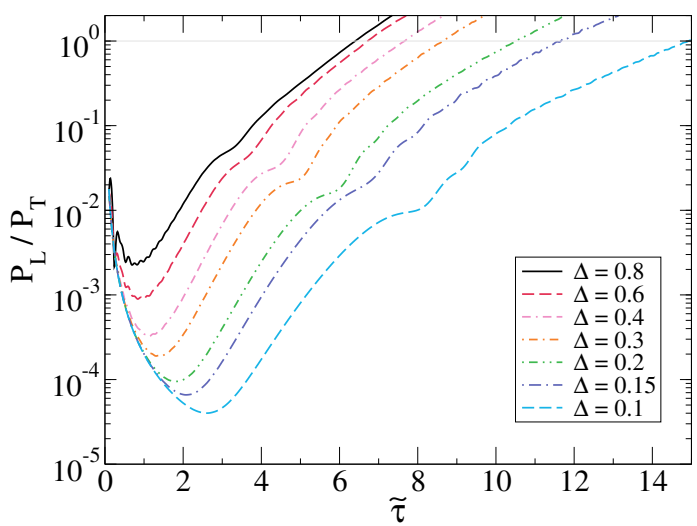

Figure 1: The left plot (a) shows the total field energy density for different initial current fluctuation magnitudes $\Delta \in\{0.1,0.15,0.2,0.3,0.4,0.6,0.8\}$. The right plot (b) features the total longitudinal pressure over the total transverse pressure as a function of proper time again for different initial current fluctuation magnitudes $\Delta \in\{0.1,0.15,0.2,0.3,0.4,0.6,0.8\}$. For both plots the data were taken from the same runs.

In Fig. 1 we present the field energy density (left) and the ratio of longitudinal to transverse pressures (right) as a function of proper time. ${ }^{1}$ From the right panel we see that after an initial transient period, there is a rapid growth in the chromofield energy density. In both plots the black solid curves are averages over 50 runs whereas the various colored dashed curves are taken from averages over 10 runs. $^{2}$ From the results contained in the right panel, one can extract the scaling of the isotropization time by extracting the proper time at which $\mathscr{P}_{L} / \mathscr{P}_{T}=1$ for the different assumed values of $\Delta$. The data shown were obtained from new runs of our simulation code which has been optimized through further parallelization so that we can efficiently investigate the dynamics at late times.

One caveat concerning the extracted isotropization times is that the HEL framework does not fully incorporate the back-reaction of the hard particles on the soft chromofields (it does so only in the limit of infinitesimally small scattering angles $\theta \sim g$, and thus is neglected in $f_{0}$ ). In addition, the hard particles are treated as unlimited energy reservoir from which the soft fields can obtain energy. For these reasons, the late time behavior of the system, when the energy density in the fields becomes comparable to that contained in the particles, is no longer reliable. Hence, one should take the inferred isotropization times as a lower bound on the time where complete isotropization could take place.

In Fig. 2 we plot the extracted isotropization times as a function of $\left(\log \Delta^{-1}\right)^{2}$. As can be seen from this figure, one finds that the isotropization time increases with decreasing initial current fluctuation amplitude. As the figure shows, we find a scaling consistent with $\tau_{\text {ISO }} \sim\left(\log \Delta^{-1}\right)^{2}$. Our data is restricted to the set of $\Delta$ 's shown in Fig. 1. As explained in Ref. [1], in order to gen-

\footnotetext{
${ }^{1}$ For LHC and RHIC initial energy densities one unit in $\tilde{\tau}$ corresponds to approximately $1 \mathrm{fm} / \mathrm{c}$ and $2 \mathrm{fm} / \mathrm{c}$, respectively.

${ }^{2}$ All runs have the lattice size $N_{T}^{2} \times N_{\eta} \times N_{u} \times N_{\phi}=40^{2} \times 128 \times 128 \times 32$ with transverse lattice spacing of $a=Q_{s}^{-} 1$ and longitudinal lattice spacing of $a_{\eta}=0.025$.
} 


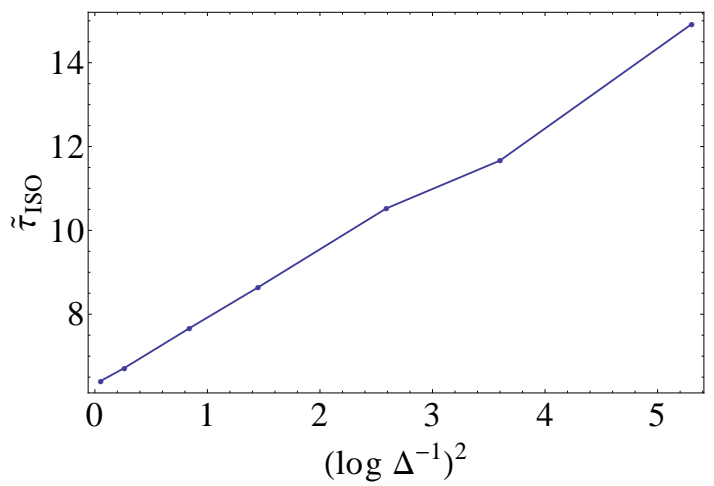

Figure 2: The isotropization time $\tilde{\tau}_{\text {ISO }}$ plotted versus $\left(\log \Delta^{-1}\right)^{2}$. The data is taken from the runs of Fig. 1.

erate gauge-field occupation numbers $\sim 1 / 2$ consistent with those expected from initial quantummechanical rapidity fluctuations [25] one should choose $\Delta \sim 1$.6. Unfortunately, due to numerical limitations stemming from the fact that we simulate compact gauge groups, we are currently unable to use such a large value of $\Delta$ and must extrapolate to the appropriate initial current fluctuation amplitude of $\Delta=1.6$. Based on the results shown in Fig. 1 and extrapolating to $\Delta=1.6$, we obtain $\tilde{\tau}_{\text {ISO }} \sim 5$.

\section{Energy Spectra}

The total longitudinal spectra are obtained following Ref. [17] by first Fourier transforming each field component $E_{\perp}\left(x_{\perp}, \eta\right), E_{\eta}\left(x_{\perp}, \eta\right), B_{\perp}\left(x_{\perp}, \eta\right)$ and $B_{\eta}\left(x_{\perp}, \eta\right)$, integrating over the transverse wave vectors and decomposing each according to the longitudinal wave vector $v$, in terms of which the electric and magnetic energy densities are decomposed into longitudinal energy spectra,

$$
\begin{aligned}
& \mathscr{E}_{E}=\int \frac{d v}{2 \pi} \mathscr{E}_{E}(v)=\int \frac{d v}{2 \pi}\left[\mathscr{E}_{E_{L}}(v)+\mathscr{E}_{E_{T}}(v)\right], \\
& \mathscr{E}_{B}=\int \frac{d v}{2 \pi} \mathscr{E}_{B}(v)=\int \frac{d v}{2 \pi}\left[\mathscr{E}_{B_{L}}(v)+\mathscr{E}_{B_{T}}(v)\right],
\end{aligned}
$$

which is numerically crosschecked by comparing with the directly calculated total energy density.

The spectral decomposition in $v$ thus defined is not gauge invariant; only the integral over $v$ is. The standard procedure to extract mode spectra is to cool down by means of Coulomb lattice gauge fixing [26, 27], which minimizes unphysical high-momentum noise within 3-dimensional time slices. However, since the left-hand-side of (4.2) is gauge invariant, the possible redistribution by means of gauge transformations of the fields is constrained, and we expect gauge dependencies to be be milder than those encountered in conventional mode spectra prior to Coulomb gauge fixing.

In order to check the robustness of the energy spectra against gauge issues we have performed random gauge transformations $\tilde{U}(x)$ which produces arbitrary noise in pure-gauge modes. The integrated energy which is manifestly gauge invariant must stay invariant under this transformation. However, because of lattice discretization there will be numerical errors in the mode spectra. We have made the amplitude of the random gauge transformations as large as possible without violating 
(a)

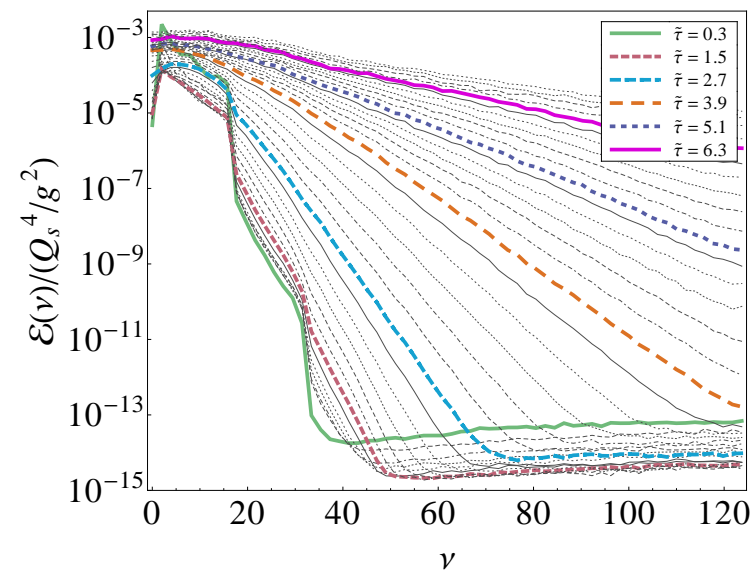

(b)

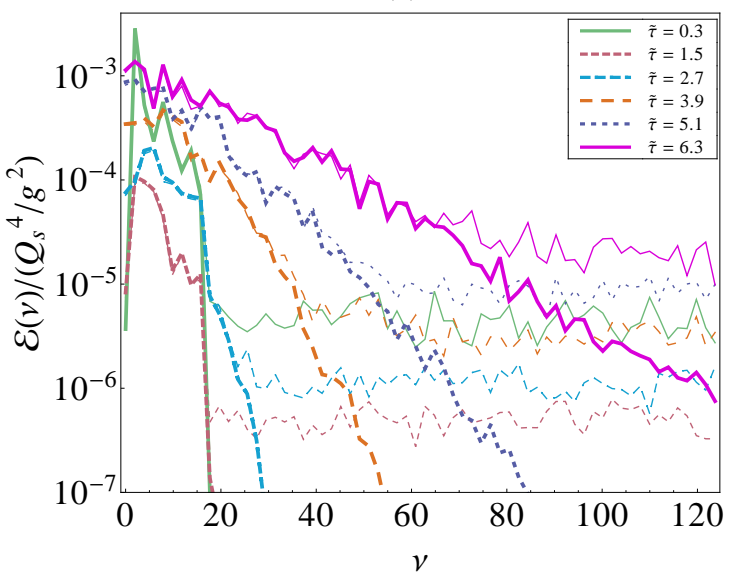

Figure 3: The longitudinal energy spectra at various proper times as a function of $v$ for the unperturbed original data (a) and the effect of a random gauge transformation on the energy spectra of a single run (b). In the latter figure thick (thin) lines give the spectra before (after) the random gauge transformation which is as strong as possible without introducing numerical errors in the total (gauge invariant) energy beyond $2 \%$.

the invariance of the total energy beyond the percent level (with occasional deviations of up to $2 \%$ ), which restricted the amplitude of the random $\operatorname{SU}(2)$ gauge parameters $\xi$ in $\tilde{U}(x)=\exp (i \vec{\xi}(x) \cdot \vec{\tau} / 2)$ to $|\vec{\xi}|_{\text {r.m.s. }} \leq 0.2$.

The left plot of Fig. 3 shows our extracted run-averaged longitudinal energy spectra at different proper times as a function of the longitudinal wave number $v$ for the original data. The right plot of Fig. 3 shows one of the individual runs before and after being subjected to random gauge transformations as described. We find that we can indeed produce a certain amount of noise in the UV part of the spectrum, while the shape of the spectrum at smaller wave numbers remains intact up to the point where it gets drowned in the UV noise. We take this as an indication that the energy spectra extracted according to (4.2) and thereby following Ref. [17] are reasonably robust for drawing physical conclusions.

However, we also found that those spectra are not completely safe — by making the amplitude of the random gauge transformations fully unconstrained-we could eventually distort the spectrum also at smaller wave numbers, but this is then accompanied by substantial violations of the sum rule provided by the gauge invariant total energy. We in fact intend to investigate gauge issues in more detail in the future by also studying the effect of gauge fixing in appropriately modified (comoving) Coulomb gauge.

As an independent check we have also analyzed the spectrum of fluctuations in the spatial distribution of the manifestly gauge invariant total energy. Besides an overwhelming zero mode, this also shows an exponential behavior in longitudinal wave number, displayed in Fig. 4a.

One can extract a longitudinal temperature by fitting the longitudinal energy density at each time step with a massless Boltzmann distribution which has been integrated over transverse momenta

$$
\mathscr{E} \propto \int d k_{z} d^{2} k_{T} \sqrt{k_{T}^{2}+k_{z}^{2}} \exp \left(-\sqrt{k_{T}^{2}+k_{z}^{2}} / T\right) \propto \int d k_{z}\left(k_{z}^{2}+2\left|k_{z}\right| T+2 T^{2}\right) \exp \left(-\left|k_{z}\right| / T\right) .
$$


(a)

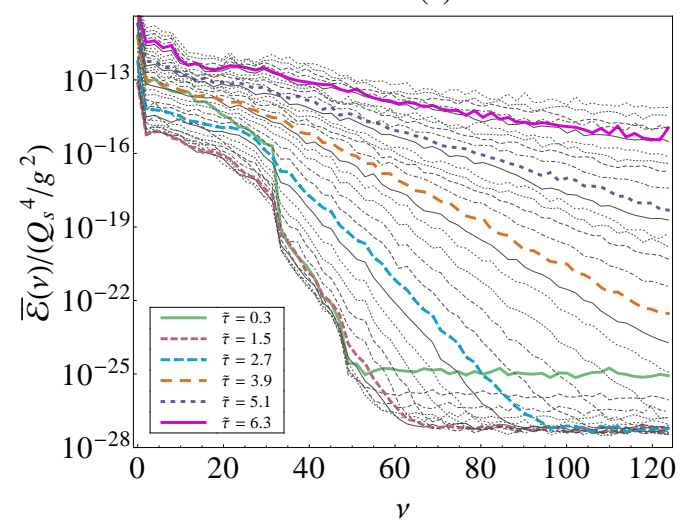

(b)

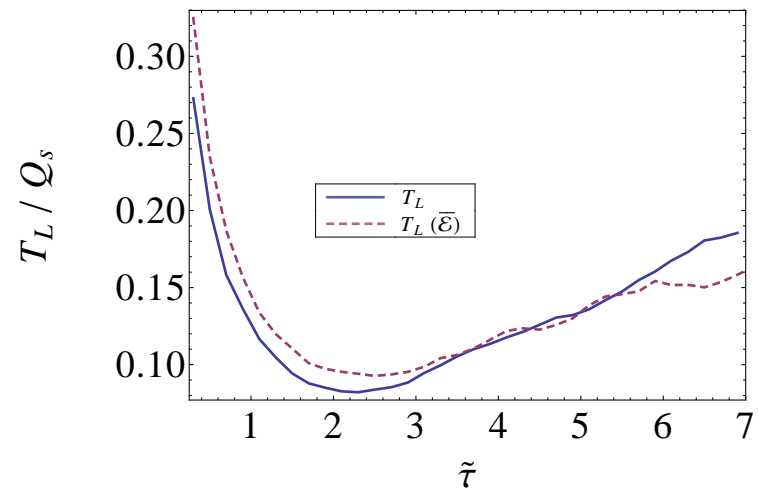

Figure 4: (a) The spectrum of spatial fluctuations $\overline{\mathscr{E}}$ in the gauge-invariant total energy as function of longitudinal wave number $v$, which besides a dominant zero-mode also shows exponential behavior; (b) The time dependent longitudinal temperature extracted from the energy spectra $\mathscr{E}(v)$ from field strength modes as shown in Fig. 3a (blue full line) and compared to that from spatial fluctuations $\overline{\mathscr{E}}$ (dashed red line).

In Fig. $4 \mathrm{~b}$ we show the evolution of the longitudinal temperature extracted from the decomposition of the energy according to the wave number $v$ in chromo-electromagnetic field strength (solid line) and compare with the one obtained from the spatial fluctuations in the gauge-invariant total energy (dashed line). The two are seen to be remarkably similar.

\section{Conclusion}

In contrast to previous simulations of anisotropic plasmas in the hard-loop regime we have found that in a free-streaming expansion there appears to be no saturation of growth of chromoWeibel instabilities and no formation of a turbulent power-law spectrum. Instead we have observed a distribution of energy in longitudinal wave numbers which is well described by a Boltzmann distribution with increasing temperature as the instabilities work towards isotropization. The latter we found to occur not before $\tilde{\tau} \sim 5$ (i.e. $\sim 10 \mathrm{fm} / \mathrm{c}$ at RHIC energies and $\sim 5 \mathrm{fm} / \mathrm{c}$ at the LHC).

In addition to these results, published in [1], we have presented new results on the scaling of the isotropization time with initial fluctuations, and we have investigated the gauge dependence of the longitudinal energy spectra extracted from Fourier modes of chromo-electromagnetic fields. While we have found the latter to be reasonably robust under random gauge transformations, and also remarkably similar to the spectrum of spatial fluctuations in the (manifestly gauge-invariant) total energy density, we intend to study the mode spectra in more detail in the future, including their form in suitably adapted Coulomb gauge so as to minimize ultraviolet noise in pure gauge modes.

\section{Acknowledgments}

We would like to thank the organizers of 'confx' and in particular Peter Arnold and Aleksi Kurkela for useful discussions, in which Peter suggested to test the robustness of our results under random gauge transformations. We thank the Vienna Scientific Cluster under project no. 70061 
and the LOEWE cluster from the Center for Scientific Computing (CSC) of the Goethe University Frankfurt under hfftheo group for providing computational resources. M.A acknowledges funding of the Helmholtz Young Investigator Group VH-NG-822. M.S. was supported by NSF grant no. PHY-1068765 and the Helmholtz International Center for FAIR LOEWE program.

\section{References}

[1] M. Attems, A. Rebhan and M. Strickland, Phys. Rev. D 87 (2013) arXiv:1207.5795 [hep-ph].

[2] U. W. Heinz, Nucl. Phys. A 418 (1984) 603C.

[3] S. Mrowczynski, Phys. Lett. B 214 (1988) 587 [Erratum-ibid. B 656 (2007) 273].

[4] Y. .E. Pokrovsky and A. V. Selikhov, JETP Lett. 47 (1988) 12 [Pisma Zh. Eksp. Teor. Fiz. 47 (1988) $11]$.

[5] S. Mrowczynski, Phys. Lett. B 314 (1993) 118.

[6] J. -P. Blaizot and E. Iancu, Phys. Rept. 359 (2002) 355 [hep-ph/0101103].

[7] P. Romatschke and M. Strickland, Phys. Rev. D 68 (2003) 036004 [hep-ph/0304092].

[8] P. B. Arnold, J. Lenaghan and G. D. Moore, JHEP 0308 (2003) 002 [hep-ph/0307325].

[9] P. B. Arnold and J. Lenaghan, Phys. Rev. D 70 (2004) 114007 [hep-ph/0408052].

[10] P. Romatschke and M. Strickland, Phys. Rev. D 70 (2004) 116006 [hep-ph/0406188].

[11] P. B. Arnold, J. Lenaghan, G. D. Moore and L. G. Yaffe, Phys. Rev. Lett. 94 (2005) 072302 [nucl-th/0409068].

[12] S. Mrowczynski, A. Rebhan and M. Strickland, Phys. Rev. D 70 (2004) 025004 [hep-ph/0403256].

[13] A. Rebhan, P. Romatschke and M. Strickland, Phys. Rev. Lett. 94 (2005) 102303 [hep-ph/0412016].

[14] A. Rebhan, P. Romatschke and M. Strickland, JHEP 0509 (2005) 041 [hep-ph/0505261].

[15] P. Romatschke and R. Venugopalan, Phys. Rev. Lett. 96 (2006) 062302 [hep-ph/0510121].

[16] P. Romatschke and R. Venugopalan, Phys. Rev. D 74 (2006) 045011 [hep-ph/0605045].

[17] K. Fukushima and F. Gelis, Nucl. Phys. A 874 (2012) 108 [arXiv:1106.1396 [hep-ph]].

[18] J. Berges, K. Boguslavski and S. Schlichting, Phys. Rev. D 85 (2012) 076005 [arXiv:1201.3582 [hep-ph]].

[19] A. Kurkela and G. D. Moore, JHEP 1112 (2011) 044 [arXiv:1107.5050 [hep-ph]].

[20] A. Kurkela and G. D. Moore, JHEP 1111 (2011) 120 [arXiv:1108.4684 [hep-ph]].

[21] K. Dusling, T. Epelbaum, F. Gelis and R. Venugopalan, Phys. Rev. D 86 (2012) 085040 [arXiv:1206.3336 [hep-ph]].

[22] P. Romatschke and A. Rebhan, Phys. Rev. Lett. 97 (2006) 252301 [hep-ph/0605064].

[23] A. Rebhan, M. Strickland and M. Attems, Phys. Rev. D 78 (2008) 045023 [arXiv:0802.1714 [hep-ph]].

[24] A. Rebhan and D. Steineder, Phys. Rev. D 81 (2010) 085044 [arXiv:0912.5383 [hep-ph]].

[25] K. Fukushima, F. Gelis and L. McLerran, Nucl. Phys. A 786, 107 (2007) [hep-ph/0610416].

[26] G. D. Moore and N. Turok, Phys. Rev. D 56 (1997) 6533 [hep-ph/9703266].

[27] A. Ipp, A. Rebhan and M. Strickland, Phys. Rev. D 84 (2011) 056003 [arXiv:1012.0298 [hep-ph]]. 